\title{
Exogeneous and Endogeneous Approaches to Semantic Categorization of Unknown Technical Terms
}

\author{
Farid Cerbah \\ Dassault Aviation - DPR/JPESA - 78, quai Marcel Dassadult \\ 92552 Saint-Cloud cedex 300 - France \\ farid.cerbah@dassault-aviation.fr
}

\begin{abstract}
Acquiring and updating terminological resources are diflicult and tedious tasks, especially when semantic information should be provided. 'I'his paper doals with Term Semantic: Categorization. The goal of this process is to assign semantic catcgories to unknown technical terms. Wo propose two approaches to the problem that rely on different knowledge sources. The exogeneous approach exploits contextinal information extracted fiom corpora. 'The endogencous approach relies on a lexical malysis of the technical terms. After describing the two implemented methods, we present the experiments tiad, we conducted on significant test sets. 'The results demonstrate that term calegorization can provide a reliable help in the terminology acepuisition processes.
\end{abstract}

\section{Introduction}

Terminological resoures have been found useful in many Ntel' applications, including Computer-Aided 'Translation and Information Retrieval. Ilowever, to latve a significant inpact on applications, terminological knowledge should not be limited to flat nomenclatures of single-word and multi-word terms. They should include semantic knowledge, such as semantic categories and various kinds of semantic: relations (lyyperonyny/hyponymy, synonymy). 'This paper focuses on the assignnent of semantic categories to technical terms. Semantic categories may play a crucial role in many applications, and particularly when disambiguation processes are recpuired. For: example, in our applicative framowork, semantic categories onsure coarse sense division of polysemous terms and are actually used to improve the French-toEnglish translation of the technical clocumentation. Assigning semantic categories to techni- cal torms is a difficult and time-consuming task. Highly specialized skills are required and, even though the major concepts represented in these terminological resources pertain to the aerondutic domain, various related knowledge arcas are concerned, such as Data Processing, Mechanics, and Mannfacturing Processes. Our goal is to claborate a tool that helps terminologists to assign semantic categories when updating the relerence terminology. Wo think that significant support can be provided to the terminologists by taking advantiage of existing catiegorized terms and their usages in documents. Such a tool can bo integrated within a terminology accquisition enviromment as a complement to a term extraction component.

Wo distinguish two kinds of approaches to semantic; caliogoriyation. In a way similas to corpus-based mothods for Word Sense Disambiguation (WSI))(Yarowski, 1992; Icle and Véronis, 1998), an exogencous approach exploits contextual information extracted from corpora in order to detomine the most plansible calcgories. By contrast, an ondogeneous approach relies solely on a lexical anlalysis of multi-word terms which are very frequent in terminological databases. This approach is based on the assumption that lexical units used in the composition of techuical terms are relevant indicators of semantic domains.

'The rest of the paper is organized as follows. Section 2 describes the teminological resources used in this study. Section 3 compares term categorization with related issues, such as thesalurus extension, WSD and term clustering. Soctions 4 and 5 describe the two proposed methods. Results and evaluation are given and discussed in section 6 . Dircetions for further rescarch are pointed out in last section. 


\begin{tabular}{|l|l|c|c|}
\hline French & English & Categories & POS \\
\hline anti-dérapage à long terme & long-action antislip & NAV & $\mathrm{N}$ \\
assiette de consigne au décollage & required takcoff attitude & CKI & $\mathrm{N}$ \\
barre de remorquage & TOO & $\mathrm{N}$ \\
dérive & tow bar & STR & $\mathrm{N}$ \\
dérive & fin & PRO, FLP & $\mathrm{N}$ \\
dériver & wander & MEC,MAI & $\mathrm{V}$ \\
dérivée & to unrivet & COM & $\mathrm{N}$ \\
embout coulissant & dorivative & FPL & $\mathrm{N}$ \\
cnrcgistreur de fatigue & sliding cndpiece & FPL & $\mathrm{N}$ \\
enregistreur de paramètres & fatiguemeter & TOO & $\mathrm{N}$ \\
jeu de protectcurs boudin cabine & flight data recorder & Det of cockpit seal protectors & $\mathrm{N}$ \\
bit d'appui touche & keystroke bit & RTL & $\mathrm{N}$ \\
amplificateur téléphone de bord & flight crew intorphone amplifier & MAI & LV \\
ne pas dóboucher & to be blind & &
\end{tabular}

- MAr: Maintenance, NAV: Navigation, CKI: Cockpit Indications, TOO: Tools, FLP: Flight Parameters, ENG: Engines, LGR: Landing Gear, STR: Aircraft Structure, DPR: Data Processing, RTL: Radiocommunications.

Table 1: A sample of the terminological database.

\section{The Terminological Resources}

We use in this study a French/English bilingual terminology of the aeronautic domain. This hand-crafted database results from a multidisciplinary effort involving technical writers, translators, terminologists and engineers. In its current state, the database contains 12,267 French/English term couples, structured in 70 semantic catcgories. As alrearly observed in sevcral terminological databases, multi-word terms cover the larger part of the database (nearly $80 \%$ ). The described terms are mostly nouns but the database also contains about 200 verbs and verbal phrases. Table 1 gives some examples of terms and a short description of the associated categories. These linguistic resources are integrated in a computer-aided translation environment used by technical writers.

Scmantic categories have originally been introduced in order to distinguish the various senses of polysemous terms. Each term couple is annotated with one or more categories specifying the contexts in which the translation is recommended. An entry is associated to a term for each identified meaning. For example, the french term dépassement has at least two possible meanings, corresponding to two different translations: overflow in the Data Processing category (DPR) and out-of-fiushness in the Aircraft Structure catcgory (STR). As shown in the examples of table 1, the assigmment of semantic categories has been extended to monoscmous terms.

In our experiments of term categorization, only the french terms have been used.

\section{Related Work}

Term Semantic Categorization is on several aspects similar to Thesaurus Extension (Uramoto, 1996; Tokunaga et al., 1997). Our methods are close to those used for positioning unknown words in thesauri. However, the two issues can be differentiated with respect to the manipulated data. A thesaurus is intended to cover a large set of conceptual domains while a terminological database is focused accurately on a specific topic and its related domains. For example, in (Tokunaga et al., 1997), the thesaurus to be extended contains more than 500 categories. This tends to make the problem harder, but, since many categories are strictly independent, it is easier to find distinctive features between categories. By contrast, our terminological database contains only 70 categories. But, in this restricted set, we find categories corresponding to close or even overlapping knowledgo areas. It is more difficult to differentiate them. 
Furthermore, the endogeneous approach, which exploits the multi-word natire of terminological units, cammot be applied to thesaurus extension because of the large amount of single-word thesanurus entriest.

It is useful to compare exogencous term categorization with corpus-based WSI methods. In both cases, contextial information extracted from corpora are used in order to assign the most plausible semantic tags to words. In WSD, the contextual cues that co-occur with the tartget word constitute the main training source whereas, in term categorization, the contextual information occurring with the term to be categorized should not be included in training data since the term is supposed to be nuknown. The only relevant training sources are the contextual cues surrounding the already categorized terms. This is a basic difference that explains why WSI) tasks usually achieve better performance than term categorization and thesaurns extensions.

In a terminology accpuisition frannework, Habert ct al. (1998) propose an exogeneous caltegorization method of unknown simple words. They use local context of simple words provided by it term extraction systom.

Burlogeneous term categorization (an also be compared with some ajproaches to term clustering (Bouriganlt and Jaceguemin, 1999; $\Lambda$ ssadi, 1997). These approaches take advantiage of the lexical and syntactic structines of techmical terms in order to build somantic chusters.

\section{Exogeneous Categorization}

Wo tested several classification models. Our first experiments were carried out with Example-based classifiers. We used our own implementation of K-nearest neighbor's algorithm $(\mathrm{kNN})$, and then the TiMBI, learner (Daclemans et al., 1999), which provides several extensions to $\mathrm{kNN}$, well-suited for NLP problems. Nevertheless, in the current state of our work, better results werc obtained with a probabilistic classifier similar to

\footnotetext{
${ }^{1}$ For Japanese, (T'okunaga et al., 1.997) reports some promising experiments of endogencous categorization to thesaurus extension. 'The approach relies on properties of Japanese word formation rules and, thus, it can hardly be adapted for other languages. Their experiments suggest that exogeneous and endogeneous approaches are complementary.
}

the one used by (I'okuniga et al., 1997) for thesamms extension. Due to lack of space, only this nothod will be deseribed in this paper.

We use as contextual cues the open-class words (nouns, verbs, adjectives, adverbs) that cooccur in the corpus with the technical terms. More precisely, the cues are open-class words surrounding the occurrences of the term in some window of predefined size. Fach new term to be ategorized is represented by the overall set of contextual cues that have been extracted from a part of the corpus (test corpus).

\subsection{Probability Model}

Let us consider a term $T$ for which the contextual cues $\left\{w_{i}\right\}_{i=1}^{n}$ have been collected in the test corpus. The categorization of this term amounts to find the category $C^{*}$ that maximizes probability $P(C \mid T)$ :

$$
C^{*}=\arg \max _{C^{\prime}} P\left(C \mid T^{\prime}\right)
$$

According to the exogeneous approach, the probability that a term $T$ belongs to catiegory $C$ depends on the contextual cues of $T$ :

$$
C^{*}=\arg \max _{C} \sum_{i=1}^{n} P\left(C \mid w_{i}\right) P\left(w_{i} \mid T\right)
$$

After applying Bayes'rule:

$$
C^{*}=\arg \max _{C} P^{\prime}(C) \sum_{i=1}^{n} \frac{P\left(w_{i} \mid C\right) P\left(u_{i} \mid I^{\prime}\right)}{P\left(w_{i}\right)}
$$

'The probabilities of the equation 3 are estimated from training datia:

- $P\left(w_{i} \mid C\right)$ is the probability that a word $w_{i}$ co-occurs with a term belonging to category $C$. It is estimated in the following way:

$$
P\left(w_{i} \mid C\right)=\frac{N_{w}\left(w_{i}, C\right)}{\sum_{w_{j}} N_{w}\left(w_{j}, C\right)}
$$

$N_{w}\left(w_{i}, C\right)$ is the number of times that $w_{i}$ co-occurs with a term belonging to category $C$.

This probability accounts for the weight of cue $w_{i}$ in category $C$. 
- $P\left(w_{i} \mid T\right)$ is the probability that $w_{i}$ cooccurs with $T$ :

$$
P\left(w_{i} \mid T\right)=\frac{N_{w}\left(w_{i}, T\right)}{\sum_{w_{j}} N\left(w_{j}, T\right)}
$$

$N_{w}\left(w_{i}, T\right)$ is the number of times that $w_{i}$ co-occurs with $T$.

- $P\left(w_{i}\right)$ is the probability of cue $w_{i}^{2}$ :

$$
P\left(w_{i}\right)=\frac{N_{w}\left(w_{i}\right)}{\sum_{w_{j}} N_{w}\left(w_{j}\right)}
$$

- $\mathrm{P}(\mathrm{C})$ is the prior probability that a term of the corpus belongs to the category $C$ :

$$
P(C)=\frac{N_{l}(C)}{\sum_{C_{i}} N_{l}\left(C_{i}\right)}
$$

where $N_{t}(C)$ is the occurrence number in training data of terms belonging to $C$. This probability accounts for the weight of category $C$ in the corpus.

\subsection{Training and Test}

The exogenoous classifier starts with the selection of test documents in the corpus. Technical terms found in these documents will form the test set. The remaining documents represent the training corpus. Training and test stages are the following:

- POS tagging. The test and training corpora are tagged with MultAna, a tagger designed as an extension of the Multex morphological analyzer (Petitpierre and Russell, 1995). Occurrences of the technical terms are identified during this stage and the terms to be categorized are those which are identified in the test corpus.

- Extraction of contextual cues. For cach term occurrence in training and test data, the contextual cues are collected. Only the lemmas of open-class words are used and cues may correspond to multiword terms. Each test term is then represented by the set of cues which have been collected in test data.

\footnotetext{
${ }^{2}$ Note that the catcgorization process could be simplified by eliminating $P\left(w_{i}\right)$, since this quantity is constant for all categories.
}

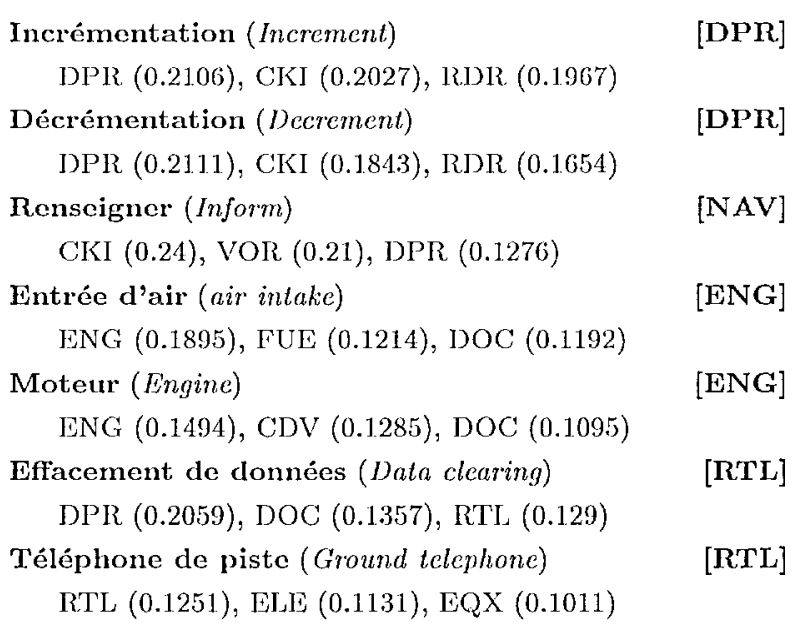

Figure 1: Some results of the exogeneous categorization.

- Frequency calculation and probability estimation. Training data are explored to compute the frequencies (occurrences and co-occurrences) of cues, terms and categories. As mentioned earlicr (section 3), the cue occurrences which have been collected around the tost terms are ignored during this step. The probabilities required for the categorization operation are then computed.

- Categorization of the test terms. The most probable categories are assigned to cach test term (sce section 4.1). Figure 1 gives some examples of exogeneous categorization ${ }^{3}$.

\section{Endogeneous Categorization}

Our approach to endogeneous categorization is simpler. It is exclusively based on a quantitative analysis of the lexical composition of tcchnical terms. Henceforth, the open-class words used to compose technical terms will be called terminological components. The endogeneous approach relies on a much more restricted source of data than the exogeneous approach, since the component set of a terminological database is quantitatively limited compared with the set of contextual cues extracted from corpora. Nevertheless, we make the assumption that this quantitative limitation is partly compensated by the

\footnotetext{
${ }^{3}$ Some category labels are described in table 1.
} 


\begin{tabular}{|c|c|c|c|}
\hline \multicolumn{2}{|l|}{ ARR } & \multicolumn{2}{|l|}{ MAP } \\
\hline architecture (architecture) & 8.440 & fonderie (casting) & 7.910 \\
\hline encadrement: (framing) & 8.440 & placage (cladding) & 7.872 \\
\hline inousse (foam) & 7.331 & schoopage (schoop process) & 7.232 \\
\hline capotiage (fairing) & 7.445 & (lrapage (laynp) & 7.111 \\
\hline châssis (ruck) & 7.353 & cuisson (baking) & 0.912 \\
\hline ćlastique (clastic) & 7.020 & moule (mould) & 6.838 \\
\hline cloison (bulkhead) & 6.632 & compacter (to compact) & 0.591 \\
\hline escamolable (retractable) & 6.441 & broche (pin) & 6.591 \\
\hline suspension (shock mount) & 6.266 & chimique (chemical) & 6.134 \\
\hline ventilation (ventilation) & 6.114 & usinage (milling) & 6.101 \\
\hline
\end{tabular}

Table 2: The top ten most significant terminological components of categories ARR (Arrangement) and MA.P (Manufacturing l'rocesses).

strong discrimination power of terminological components.

The training phase assigns to cach category a set of representative components with respect to some association scorc. The categorization phase determines the most plausilule categories of a term according to its components.

\subsection{Association Score}

To estimate the dependency between components and categories, we experimented several association criteria. The choice of these criteria has been influenced by the comparative stucly described in (Yang and Perdersen, 1997) on feature seloction criteria for text catogorization. Wo tested several moasures, including component frecuency, information gain and nutual information. Our best resultis were achieved with mut,ual information which is cstimated nsing:

$$
I(w, C) \approx \log _{2} \frac{N_{w}(w, C) \times N_{w}}{N_{w}(C) \times N_{w}(w)}
$$

- $N_{w}(w, C)$ is the frecuency of component $w$ in catcgory $C$.

- $N_{w}$ is the total number of component occurrences.

- $N_{w}(C)$ is the total number of component occurrences in category $C$. This factor reduces the effect of the compononts weakly represented in category $C$, compared with the other components of $C$.

- $N_{w}(w)$ is the frequency of component $w$ in the terminological clatabase. T'his factor reduces the effect of the components that denote basic concepts spread all over the database. For example, the components speed, altitude, pressure have high freguen(cies in category ILP (Flight Parametors), but, as basic concepts, they also appear frequently in many categories.

Table 2 gives for two categories the ten most representative components according to this score.

'The association score between a term $T$ ' (with components $\left\{u_{i}\right\}_{i=1}^{n}$ ) and a categrory $C$ is given according to the components of $T$ :

$$
A_{l}(T, C)=\frac{N_{l}(C)}{N_{l}} \sum_{i=1}^{n} I\left(w_{i}, C\right)
$$

$N_{t}(C)$ is the number of terms pertaining t, (a) categery $C$ and $N_{t}$ is the total number of terms. 'The factor $\frac{N_{t}(C)}{N_{t}}$ favors larger cattegories.

The categorization task determines the category $C^{*}$ that maximizes the association score:

$$
C^{*:}=\arg \max _{C} A_{t}(T, C)
$$

\subsection{Training and Test}

Only multi-word terms can be categori\%ed with this method since our endogeneous approach is by nature not relevant for simple words. A test set of compound terms is extracted from the terminological database. The remaining terms are used for training. The training terms are analyzed in order to assign to each category its terminological components. Then, component frequencies and association scores are computed. 


\begin{tabular}{cccccc}
\hline \# $\mathrm{T}$ & $\mathrm{k}=1$ & $\mathrm{k}=2$ & $\mathrm{k}=3$ & $\mathrm{k}=4$ & $\mathrm{k}=5$ \\
\hline 312 & 45.07 & 68.22 & 79.15 & 88.10 & 91.40 \\
89 & 42.25 & 73.14 & 86.95 & 91.57 & 98.19 \\
98 & 50.65 & 84.94 & 91.11 & 94.93 & 96.96 \\
120 & 48.11 & 78.95 & 91.26 & 96.83 & 97.92 \\
253 & 59.17 & 81.98 & 92.35 & 96.75 & 98.65 \\
125 & 73.16 & 89.30 & 96.85 & 98.68 & 99.59 \\
234 & 68.19 & 87.63 & 94.14 & 96.99 & 98.31 \\
205 & 49.05 & 83.86 & 95.12 & 98.61 & 99.47 \\
203 & 47.25 & 72.18 & 85.66 & 94.40 & 98.48 \\
105 & 44.55 & 69.61 & 87.28 & 93.97 & 97.11 \\
\hline Tot. 1744 & & & & & \\
Avg. & 52.75 & 78.98 & 89.99 & 95.08 & 97.61 \\
\hline
\end{tabular}

Table 3: Results for the exogencous approach. 'Ten experiments have been run for a total test set of 1744 terms.

\begin{tabular}{lccccc}
\hline \#T & $\mathrm{k}=1$ & $\mathrm{k}=2$ & $\mathrm{k}=3$ & $\mathrm{k}=4$ & $\mathrm{k}=5$ \\
\hline 229 & 47.96 & 62.05 & 71.23 & 75.77 & 78.50 \\
232 & 42.60 & 56.38 & 62.28 & 69.73 & 74.23 \\
228 & 41.5 & 57.89 & 63.03 & 72.76 & 76.27 \\
227 & 45.54 & 61.05 & 68.57 & 76.74 & 80.18 \\
239 & 46.38 & 61.01 & 67.93 & 73.41 & 75.94 \\
229 & 43.41 & 57.54 & 65.42 & 71.16 & 75.34 \\
231 & 43.75 & 60.74 & 70.04 & 73.97 & 77.62 \\
228 & 42.85 & 60.18 & 66.97 & 70.50 & 73.85 \\
237 & 45.68 & 58.62 & 66.37 & 72.10 & 74.67 \\
240 & 42.30 & 58.82 & 68.48 & 70.29 & 74.05 \\
\hline 'Iot. 2320 & & & & & \\
Avg. & 44.19 & 57.77 & 67.03 & 72.64 & 76.06 \\
\hline
\end{tabular}

Table 4: Results for the endogeneous approach. Ten experiments have been run for a total test set of 2320 terms.

During the test phase, cach test term is annotated with the most plausible categories according to its components.

\section{Experiments and Evaluation}

To estimate the accuracy of the exogeneous method, we used a domain-specific corpus of 541,964 words, composed of documents pertaining to various textual genres (software specifications, maintenance procedures, manufacturing notices...). This corpus covered 63 of the 70 categories. Each run starts with the selection of a document among the corpus documents. The known terms identified in this document are considered as test terms. We used relatively wide contexts. The cues were extracted in a window of \pm 20 words around the term. Each rum involved more than 70,000 contexts of term occurrences. To experiment the endogencous approach, test sets of compound terms have been randomly extracted from the terminological database.

We adopted an evaluation scheme similar to that defined in (Tokunaga et al., 1997) for thesaurus extension. The categorization is considered successful if the right, category appears among the $k$ first categories assigned by the classifier. Within a semi-automatic acquisition. framework, this evaluation scheme is more suitable than strict evaluation where only the first category assigned by the classifier is considcred as relevant (evaluation restricted to $k=1)^{4}$. From our application perspective, it is useful to provide to the terminologist a restricted set of less than 5 plausible categories instead of the complete set of 70 categories without prior filtering.

In the experiments described in (Tokmuga et al., 1997), $k$ : takes the values $5,10,20$ and 30 for averaged performance ranging from $26.4 \%$ to $55.9 \%$ (the choice is made among 544 categories). Some of their precise experiments yielded an accuracy greater than $80 \%$ for $k$ : $=30$. In our experiments, we measured accuracy for $k=1$ to 5 . Some results are given in tables 3 and 4 . The scores are higher than those achieved in thesaurus extension, especially with the exogeneous approach (from $52.75 \%$ to $97.61 \%$ ). We should however keep in mind that we deal with a different kind of data (sce section 3 ).

\section{Conclusion and Further Work}

We have presented in this paper two approaches to term semantic categorization that have been fully implemented and experimented on significant test sets. The results achieved in this work demonstrate that term categorization tasks could be integrated within a semi-automatic

\footnotetext{
${ }^{4}$ Some limitations of strict evaluation are also pointed out in (Resnik and Yarowski, 1999).
} 
terminology acquisition process to provide an active support to terminologists.

The solutions to this problem can be considerably improved and we have identified several promising directions for furthes research.

Our experiments show that exogeneous catigorization is noticcably the most efficient of both approaches. However, it requires much more knowledge sources and computational overhead. It is more exposed to data sparseness, since large amounts of contextual datia are not always available, especially in technical domains. Wo should stress that this study benefited from the availability of a highly relevant corpus. This meins that, for sake of robustiness, other methods (even less efficient) and relevant knowledge sources should not be neglected. The two proposed approaches are conpelenentary in the sense that they take atvantage of distinct knowledge souress. Further work will investigate the various ways to combine them in order to improve the overall performanece.

The use of relational information, and particularly syntactic relations, is another major: direction for further rescarch. Hxogencous catcegorization is based on a bag of words/Jommas model since wide contexts of lemmatized words were nsed, without, consideration for the positions of these canes and their potential syntactic relationships with the target terms. Syntactic information cxtracted from local context, as verb-object relations, is mother major somree for: exogeneous categorization that has been exploited in thesaurus extension methods. The endogeneous approach can also be improved by exploiting the syntactic structine of the technical terms. In our approach, all components of technical terms are equally weighted, independently of their syntactic roles within the terms. More accurate association scores can be introduced by taking advantage of head/modifier relations.

Finally, we should note that the bilingual nature of our terminological resources has not been taken into account. Minor changes are required to make the two classifiers work for Engrish. Futher experiments will be conducted on the English resources. In this bilingnal context, cither the French or the English expression (or both) could be used to categorize a given term.

\section{References}

H. Assadi. 1997. Knowledge Acquisition from 'Texts : Using an Autonatic Clustering Method Bassed on Nom-Modifier Relationships. In 35th Annual Mecting of the Association for Computational Linguistics, Maurid.

D. Bouriganlt and C. Jaccuenin. 1999. Term Extraction + 'T'erm Clustering: An Integrated Platform for Computcr-Aided Terminology. In Proceedings of the European Chapter of the Association for Computational Linguistics (EACL 99), pages 15-22, Bergen.

W. Daclemans, J. Zavrel, K. van del Sloot, and Antial van den Bosch, 1999. TiMBL: Tilburg Memory I3ased Learner - Version 2.0 -.. Refercence Guide. 'Tilburg University.

B. Habert, A. Nazarenko, P. Zweigenbaum, and J. B3onaud. 1998. Extonding an Existing Specialized Somantic; Lexicon. In Proceedings of first International Conference on Language Resources and Holuation, pages 663- 668 , Granada.

N. Ide and .J. Véronis. 1998. Introduction to the Special Issue on Word Sonse Disambigitation: 'The State of the Art. Computational Listguistics, 24(1): 1-40.

1). P'etitpierre and G. Rnissell. 1995. MMorpli The Multext Morphology Program. Technical report, Multext Deliverable 2.3.1.

P. Ressnik and D. Yarowski. 19999. Distinguishling systems and distinguishing sonses: new evalnation methods for Word Sens Disannbignation. Natural Language Enginecring, 5(2):113 133.

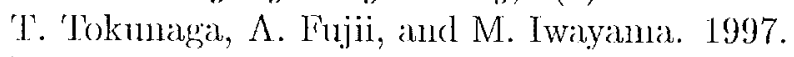
Extending a thesinurus by classifying words. In Association for Computational Jinguistics, editor, ACL Workshop on Automatic Information Jixtraction and Building of loxical semantic Resourees, pages 16-21.

N. Uramoto. 1996. Positioning unknown words in a thesaurus by using information extracted from a corpus. In Proceedings of Coling 96, pages 956-961.

Y. Yang and J. Perdersen. 1997. A comparative study on feature selection in text categorization. In Procedings of the Fourteenth Intentrational Conference on Machine Learning (ICML'97),. 1). Yarowski. 1992. Word Sense 1)isambignat tion Using Statistical Models of Roget's Catcgories Trained on Large Corpora. In Procedings of Coling 92, Nantes. 